\title{
Comunicar
}

\section{Un proyecto IM/CC que estimula pensamiento y cooperación}

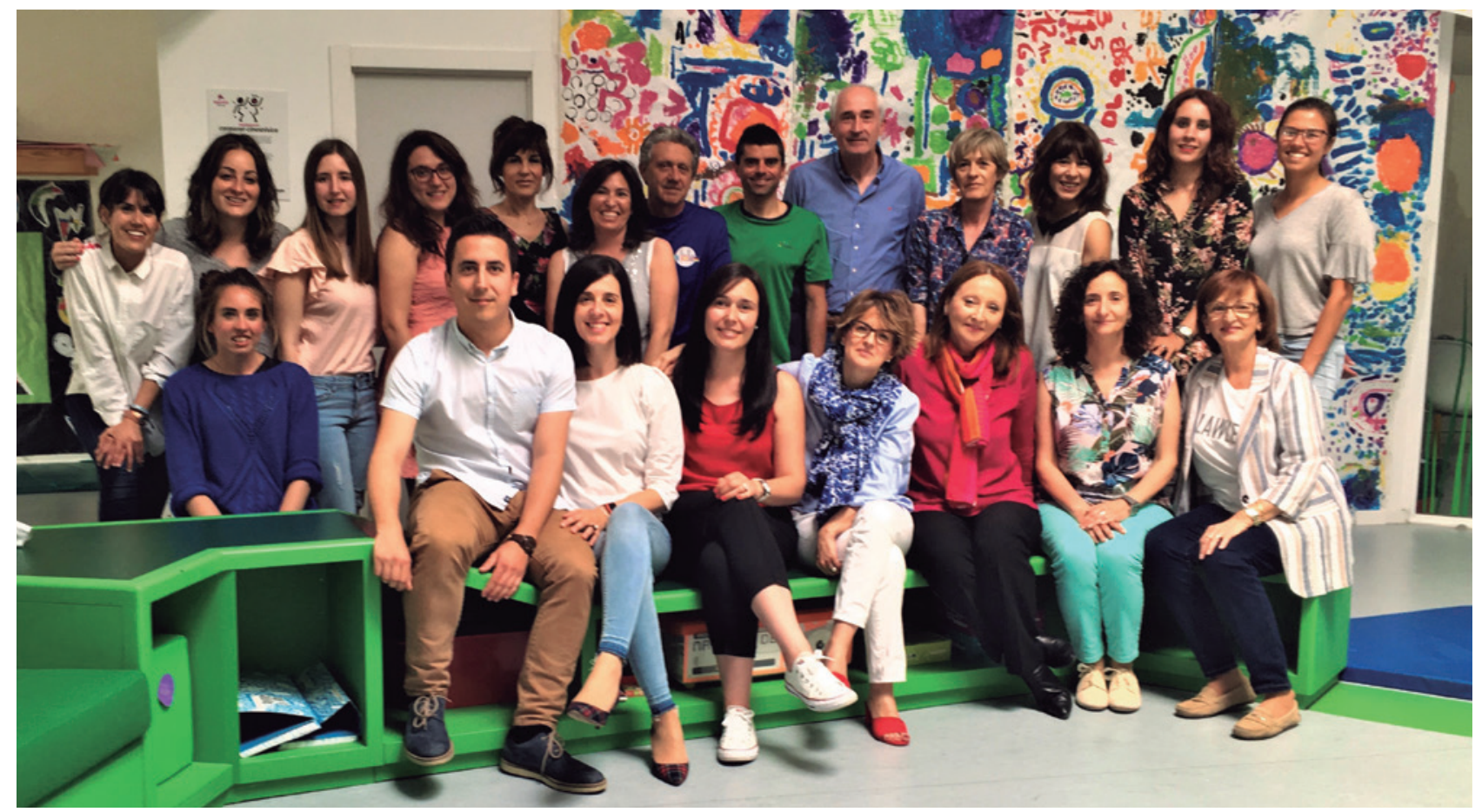

El desarrollo del enfoque didáctico de las inteligencias múltiples es clave para potenciar todos los medios de representación y expresión con todos los modelos y formatos de pensamiento.

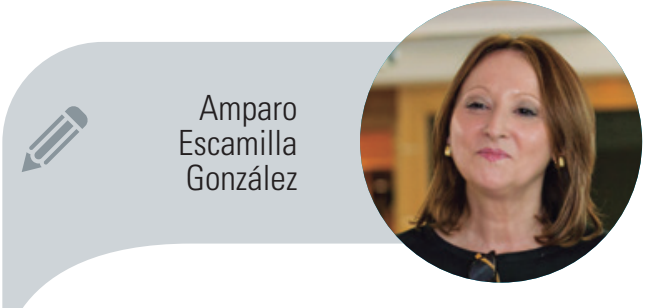

Directora de innovación en el Liceo Europa de Zaragoza proyectospedagogicos@gmail.com

Por ello, resulta extraordinariamente interesante y atractivo, convertir la propia comunicación y sus medios en el objeto específico de atención de un proyecto que persigue avanzar en la construcción de una personalidad más autónoma, crítica y cooperativa. 


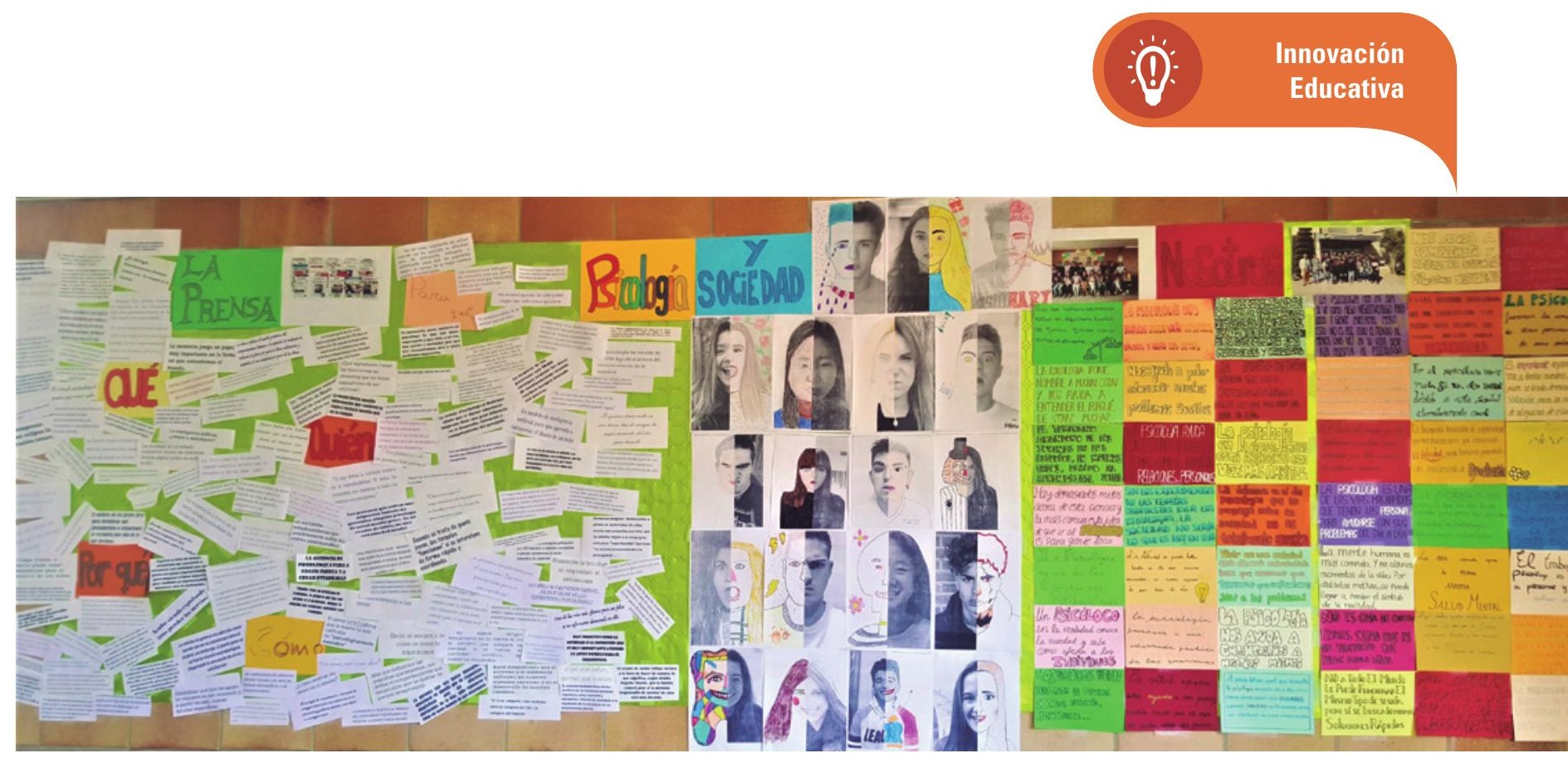

investigado y generado alternativas para enriquecer la propuesta.

Vinculado a un proyecto que esta etapa de infantil tenía como propio (Inventos e inventores), hemos puesto en práctica situaciones en las que equipos de alumnos y alumnas de primaria han explicado, apoyándose en técnicas de pensamiento y cooperación, algunos de los objetos e inventos relacionados con la comunicación y sus inventores. Por otro lado, las visitas a las exposiciones de productos de primaria han resultado muy motivadoras para los niños y niñas de infantil, que aprovechan para explorar espacios, aumentar lazos de relación y convivencia, resolver interrogantes y plantearse otros muchos.

En Educación Secundaria Obligatoria se han materializado también algunas líneas de desarrollo del significado de Comunicar. Alumnos y alumnas de distintos cursos, asesorados por el director general, Manuel Zapater, han participado en el desarrollo de elementos de trabajo vinculados a la oratoria, generando podcast muy interesantes para comunicar y expresar resultados de una investigación que han hecho relacionada con los efectos de algunos medicamentos.

En Bachillerato, el proyecto de los alumnos de Psicología de segundo, Ilevado de la mano de María Jesús Hernández, se propuso como meta analizar cómo la prensa escrita refleja la aportación de la psicología a la sociedad.

Siguiendo la técnica de pensamiento del análisis asociativo, cada alumno ha trabajado sobre nueve artículos de su elección, publicados en tres momentos distintos a lo largo del curso y que trata-

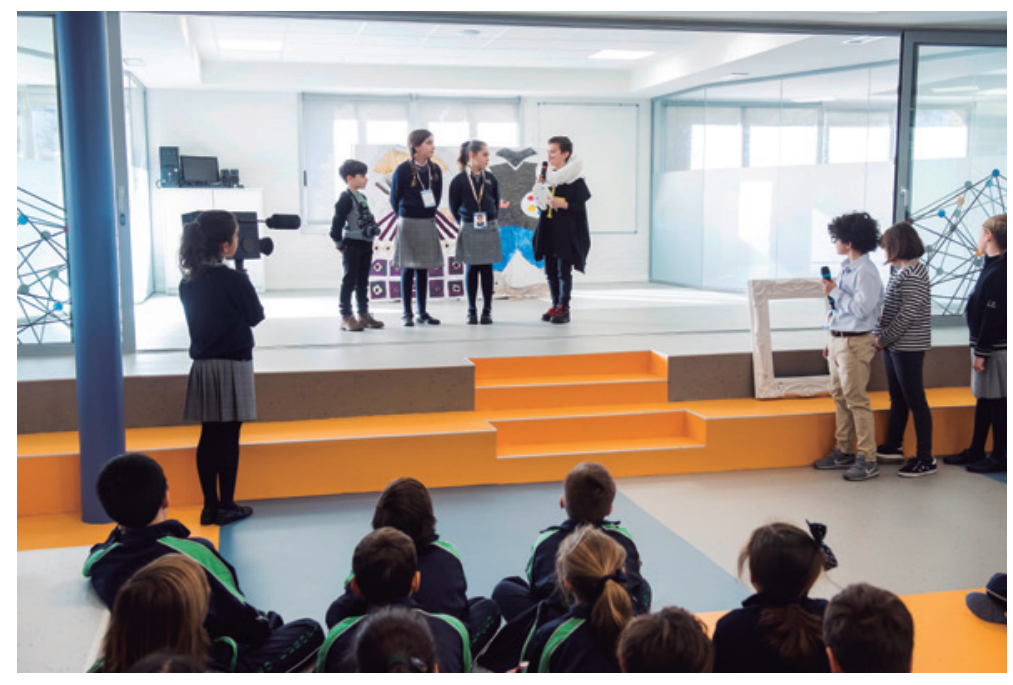

\section{Buscamos el diálogo integrador}

\section{entre líneas y estrategias}

\section{de innovación en materias y}

\section{elapas}

ban sobre el ejercicio profesional de la psicología en distintas áreas. En una primera fase, los alumnos analizaron cada artículo siguiendo un esquema propuesto y en una segunda fase elaboraron un informe con sus conclusiones.

Para finalizar, se realizó un mural con las frases más significativas de los artículos y de las valoraciones. El proyecto se completó incorporando al mural un ejer- 


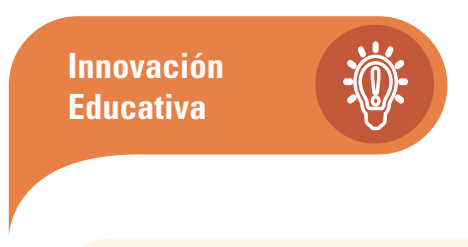

\section{Inteligencia visual-espacial.} estética de los textos que estáis construyendo y explica las razones que aportas.

1 Valora la composición espacial y estética de las obras plásticas que has elaborado sobre tu medio y explica las razones que aportas. CEC. SIEP CM-CCT

- Valora la composición espacial y
- Construye con tu equipo un anuncio publicitario relacionado con tu medio que podría ser incluido en el periódico de primaria.

- Prepara exposiciones orales de tu medio o un profesional para presentar en público aplicando una técnica de pensamiento y rúbrica de la comunicación oral.

\section{Inteligencia lingüística. CCL}

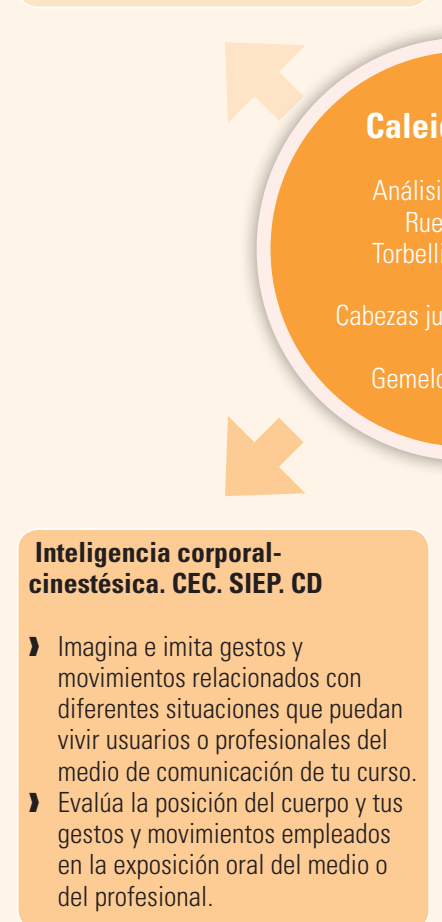

rando continuamente intercambios entre aulas, cursos, ciclos y etapas. Los alumnos se implican en experiencias de intercambio en las que asumen roles de profesores y mentores. Las familias cooperan para apoyar, informar y transmitir conocimientos relacionados con los temas tratados.

Globalmente los principales trabajos guiados por los caleidoscopios IM/CC técnicas de pensamiento y cooperación (para trabajar con los lenguajes y recursos de todas las inteligencias) han sido:

त En el primer trimestre: todos los cursos profundizaron en su medio (teléfono, carta, radio, televisión, prensa e internet). Plasmaron el contenido en mapas visuales en castellano, inglés y francés (dos por curso) que realizaron en equipos cooperativos y después sintetizaron en propuestas de curso. En ellos se trabajaba el estudio de un medio y de un profesional del medio con una rueda lógica y un análisis asociativo. Para construir los contenidos, las técnicas de pensamiento se ejercitaron con técnicas cooperativas (gemelos pensantes, folio giratorio, cabezas juntas numeradas, 1-2-4). Cada curso construyó la maqueta del edificio de su medio, que formaba parte de la ciudad de la comunicación. Definieron y solucionaron problemas económicos, sociales y culturales vinculados a los medios. Elaboraron decálogos de uso y disfrute construido de cada uno de ellos. Seleccionaron canciones y melodías. Dramatizaron y expresaron, con gestos, movimientos y palabras, situaciones comunicativas personales y sociales. Ayudaron y se apoyaron entre cursos (por ejemplo, 5. ${ }^{\circ}$ curso que investigó la prensa escrita, preparó materiales y explicó a segundo curso — que estudiaba el correo- el significado de la sección periodística "Cartas al director"). Elaboramos vídeos con las fotografías del proceso y se preparó una gran exposición con los mapas mentales de las técnicas de pensamiento, la maqueta de la ciudad de la comunicación y los decálogos de uso y disfrute constructivo de cada uno de los medios. 


\section{Técnicas de pensamiento \\ y cooperación comunes se}

\section{aplican en todas las etapas}

\section{que participan en el proyecto}

7 En el segundo trimestre, se llevaron a cabo los estudios de evolución de cada medio, vinculados a sucesos sociohistóricos relevantes y se construyeron grandes líneas del tiempo para documentarlo e ilustrarlo. Se hicieron intercambios continuos entre alumnado y grupos para compartir lo investigado en cada curso y se llevaron a cabo experiencias de mentoría muy interesantes, como la de 6. ${ }^{\circ}$ curso con 2..: los mayores ayudaron sus compañeros y compañeras de $2{ }^{\circ}$ curso a escribir cartas, muy ilustrativas y motivadoras, a grandes personajes históricos.

خ En el tercer trimestre, además de ir completando obras plásticas y trabajos lógico-matemáticos, se impulsaron las prácticas de dramatización y lenguaje para grabar vídeos en los cuales se explicaban las líneas del tiempo. Se ilustraron grandes tabloides de prensa y se construyó un periódico muy especial: cada curso aportaba trabajos de tres géneros periodísticos (noticias, columnas, reportajes, entrevistas, etc.) en los cuales la información giraba sobre su propio medio (subidas en los contratos telefónicos, la incidencia del correo electrónico, la programación televisiva, las retransmisiones deportivas por radio, etc.). Se preparó una gran exposición integrando los trabajos de los tres trimestres (mapas mentales y visuales, líneas del tiempo, cartas al director, cartas a personajes históricos, periódico editado en material impreso - algunos ejemplares en DIN A2 que después preparamos en formato elec-
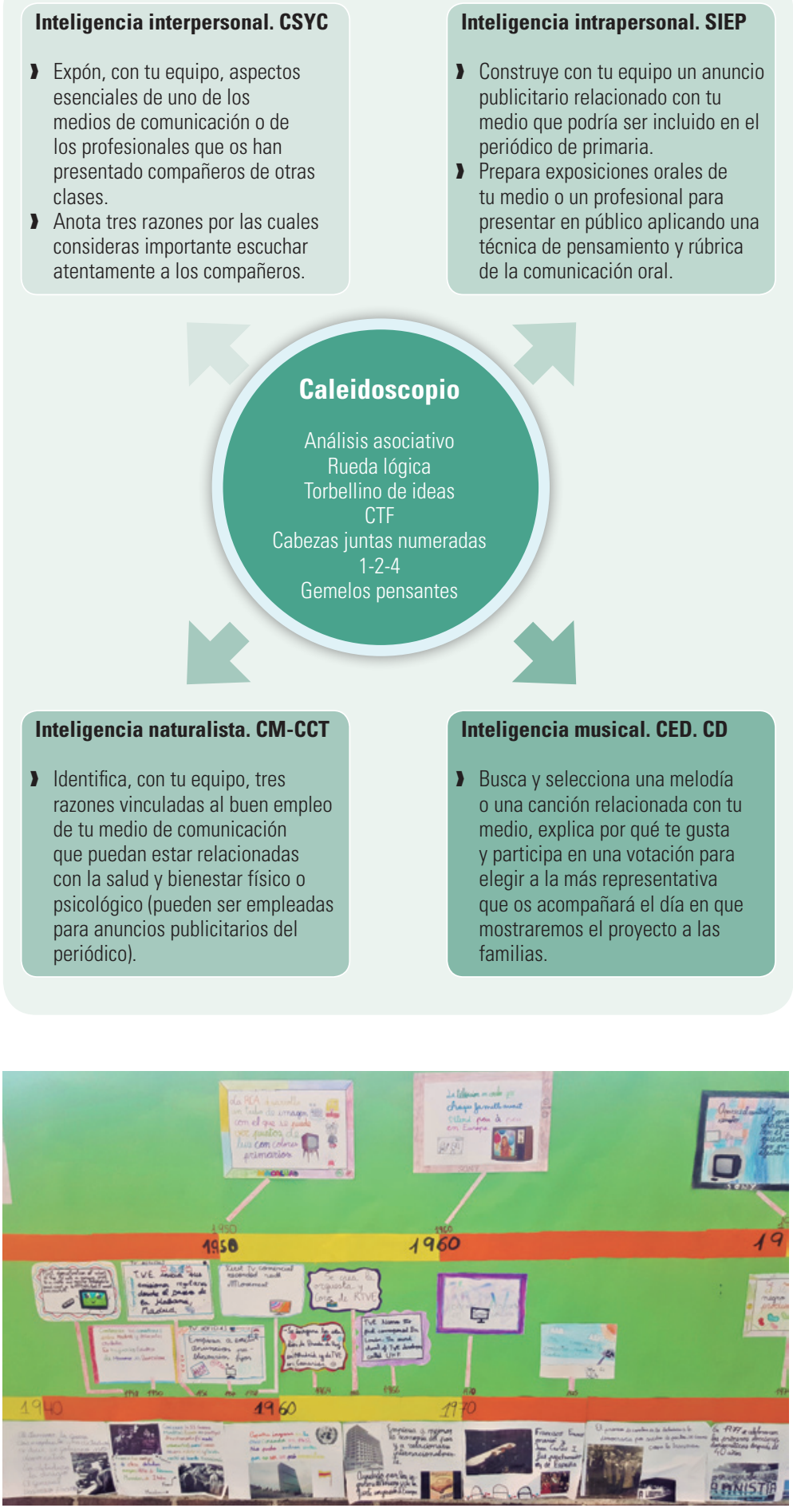

trónico para que las familias lo pudieran descargar y conservar-). Alumnos y alumnas de primaria e infantil explicaron los hitos del trabajo realizado y, finalmente, las familias disfrutaron con los vídeos grabados con los niños disfrazados presentando las líneas del tiempo y algunas otras situaciones comunicativas relacionadas con una performance de un proyecto 


\section{ACTIVIDADES DE AULA}

Muchas situaciones de trabajo de cualquiera de las etapas van a requerir construir pensar y dialogar sobre temas como la comunicación, los medios y sus profesionales. Sugerimos construir estos temas tan significativos de manera activa, fomentando la reflexión y los intercambios por medio de técnicas de pensamiento y cooperación.

Así, por medio de una rueda lógica y un folio giratorio:

1. ¿Qué es comunicar? ¿Qué tipos de comunicación conoces? ¿Qué medios de comunicación social conoces?

2. ¿En qué se parecen y en qué se diferencian la comunicación en los animales y en las personas? ¿En qué se parecen y qué se diferencian la comunicación en prensa escrita y televisión?

3. ¿Por qué es imprescindible comunicar? ¿Qué repercusiones tiene en nuestra vida?

4. En síntesis, ¿qué es comunicar y qué nos aporta?

Y por medio de un análisis asociativo y un cabezas juntas numeradas podemos dialogar y pensar sobre el periodista de medios impresos y digitales. ¿Dónde trabaja un periodista, dónde investiga? ¿Cuándo lo hace? ¿Por qué lo hace? ¿Qué medios emplea? ¿Quiénes le ayudan y con qué? ¿Qué normas ha de aplicar en su trabajo?
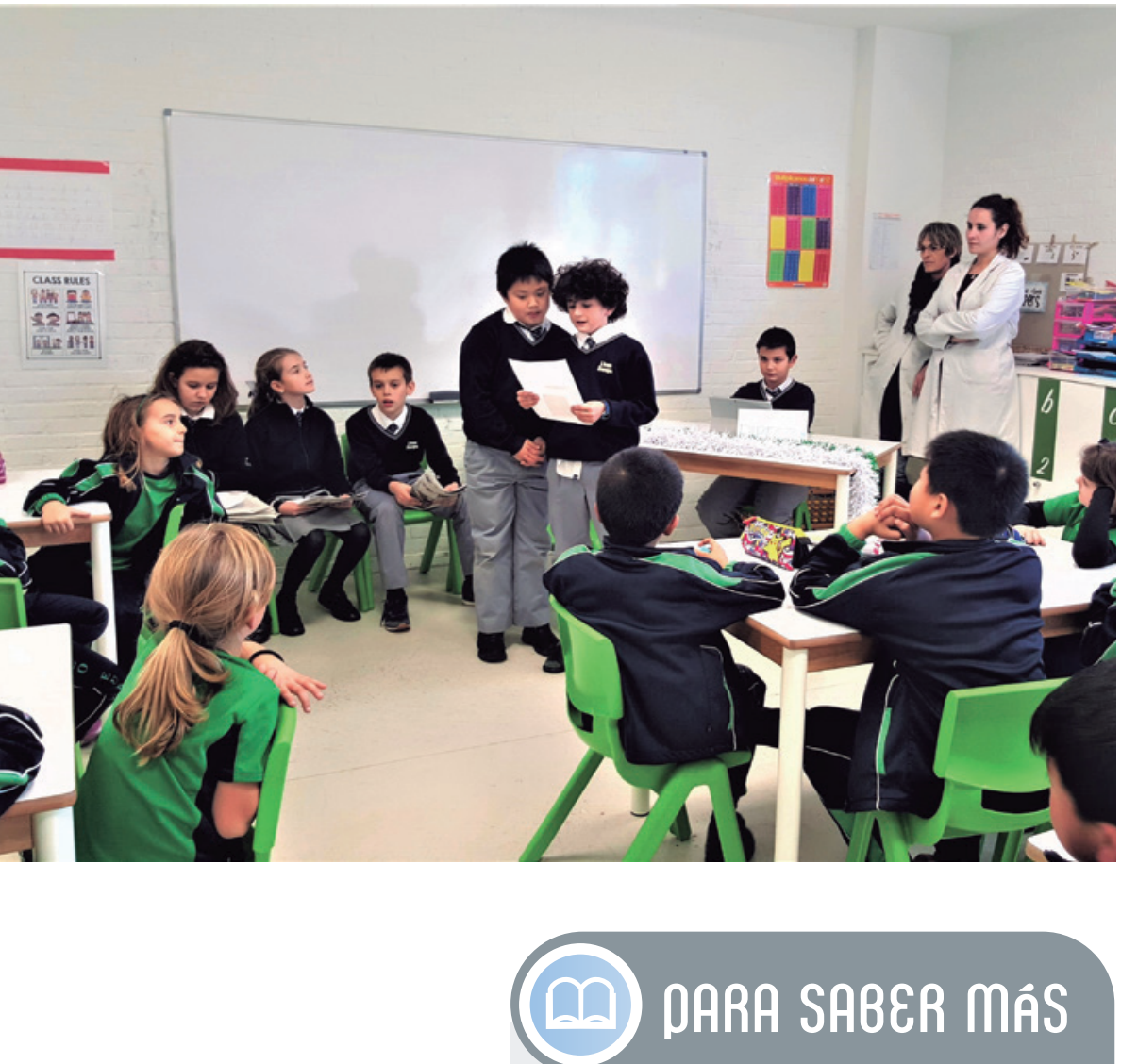

Escamilla, A. (2020). Inteligencias múltiples en la práctica. Nuevas estrategias y nuevas herramientas. Barcelona: Graó.

Escamilla, A., Fuentes, M. I., y Pinto, S. (2016). Retos. Guías Didácticas. Madrid: Anaya.

Moruno, P., y VARAS, M. (2016). El aprendizaje cooperativo. Madrid: Anaya. de Plástica con retratos y personajes representados por Goya.

\section{Evolución en los proyectos}

Después de estos tres años de dinámicas de trabajo tan ricas y estimulantes, resulta muy gratificante constatar:

入 Cómo el profesorado impulsa los procesos de construcción del pensamiento analítico, lógico, crítico, creativo y metacognitivo en el desarrollo de estos programas estratégicos.

入 Cómo se integran las experiencias de aprendizaje cooperativo (gemelos, folios giratorios, cabezas juntas numeradas, 1-2-4) con las técnicas de pensamiento.

入 Cómo se aplican los dos tipos de técnicas, de manera natural, para trabajar emociones (la admiración, el asombro, la alegría, entre otras) con valores (el compañerismo, el esfuerzo, entre otros).

$\boldsymbol{\lambda}$ Cómo alumnos y alumnas se muestran cada vez más autónomos, cooperativos, críticos y creativos.

त Cómo disfrutan y se sorprenden las familias observando los productos tan trabajados que expresan tanto entusiasmo e implicación.

Seguiremos avanzando, investigando y compartiendo nuestras inquietudes y nuestros trabajos. Es una necesidad y es una gran fuente de motivación explorar nuevas líneas de trabajo y considerarlo una vía de crecimiento indispensable en esta nuestra maravillosa profesión •

\section{(-) hemOS HABLADO DE \\ Inteligencias múltiples; competencias clave; proyectos; aprendizaje basado en problemas; técnicas de pensamiento; técnicas de cooperación.}

Este artículo fue solicitado por PADRES Y MAESTROS en marzo de 2020, revisado y aceptado en junio de 2020. 